\title{
Adolf Jülicher als Paulusforscher - anläßlich seines 150. Geburtstages
}

\author{
(gehalten in der Plenarsitzung am 26. Januar 2007)
}

\author{
OtTo Merk
}

Adolf Jülichers an seinem heutigen 150. Geburtstag zu gedenken, heißt, anhand der Persönlichkeit eines Gelehrten, seines Wirkens und Forschens, einer bedeutenden Epoche theologischen Denkens, der „liberalen Theologie“, in einem begrenzten Ausschnitt zu begegnen.

\section{Doch wer war (Gustaf) Adolf Jülicher? ${ }^{1}$} Der am 26. Januar 1857 in Falkenberg bei Berlin Geborene wurde nach Schulzeit und Studium in Berlin 1880 in Halle mit einer alttestamentlichen Untersuchung zum Lic. phil. promoviert. ${ }^{2}$ Er erwarb 1886 in Berlin mit einem Werk über die Gleichnis

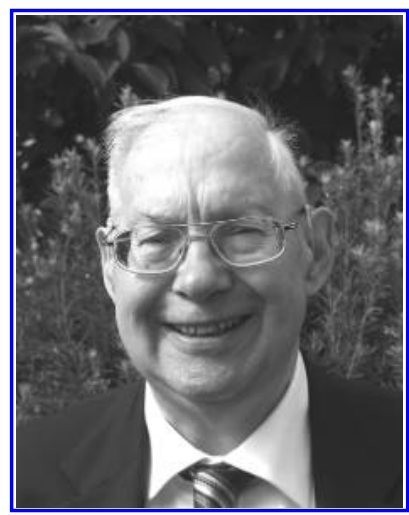

Otto Merk, Professor für Neues Testament an der Universität Erlangen, K. Mitglied der Göttinger Akademie seit 2006

Zur reichen Literatur zur Person und über das Werk vgl. in Auswahl: A. Jülicher in seiner Selbstdarstellung, in: Die Religionswissenschaft der Gegenwart in Selbstdarstellungen, hg. v. E. Stange, Bd. IV, 1928, 154-200 [abgek.: A. Jülicher; zitiert gemäß Doppelpaginierung S. 1-41]; H. v. Soden, Akademische Gedächtnisvorlesung für Adolf Jülicher, ThBl 18, 1939, 112; N. van Bohemen, Art. Jülicher, Adolf, DBS IV, 1949, 1414-1417; W.G. Kümmel, Adolf Jülicher (1857-1938) - Theologe, Neutestamentler und Kirchenhistoriker, in: ders., Heilsgeschehen und Geschichte, Bd. 2. Ges. Aufs. 1965-1976, hg. v. E. Gräßer u. O. Merk, MThSt 16, 1978, 232-244 (Lit.); H.-J. Klauck, Adolf Jülicher - Leben, Werk und Wirkung, in: Historische Kritik in der Theologie. Beiträge zu ihrer Geschichte, hg. v. G. Schwaiger, Studien zur Theologie- und Geistesgeschichte des Neunzehnten Jahrhunderts, Bd. 32, 1980, 99-150 (mit fast vollst. Bibliographie); O. Merk, Jülicher, (Gustaf) Adolf, in: Literaturlexikon (Hg. W. Killy), Bd. 6, 1990, 150f.; B. Reicke, Jülicher Adolf (1857-1938) in: DBI, Vol. 1: AJ, 1999, 650f.; J.-Chr. Kaiser, Adolf Jülicher als Zeitgenosse. Eine biographische Skizze, in: U. Mell (Hg.), Die Gleichnisreden Jesu 1899-1999. Beiträge zum Dialog mit Adolf Jülicher, BZNW 103, 1999, 257-286 (Lit.); W. Baird, History of New Testament Research, Vol. 2: From Jonathan Edwards to Rudolf Bultmann, 2003, 156-163 u.ö. - Weiter vgl. die bibliographischen Angaben bei H. Schmidt, Art. Jülicher, Adolf 1857-1938, in: ders., Quellenlexikon zur deutschen Literaturgeschichte, Bd. 15, 1998, 108. 
reden Jesu den Dr. theol; 1887 erfolgte dort mit einer patristischen Arbeit die Habilitation in Kirchengeschichte. 1888 aufgrund eines fulminanten Gutachtens von A. Harnack aus dem Pfarramt (1882-1888) nach Marburg auf eine a. o. Professur berufen, war er dort von 1889 bis zu seiner Emeritierung im Jahre 1923 o. ö. Professor für Neues Testament und Alte Kirchengeschichte. Über diese „Umgrenzung“ seines Lehr- und Forschungsbereichs war Jülicher stets sehr befriedigt, denn - so schreibt er - „das Neue Testament und die alte Kirchengeschichte gehören zusammen, und besonders dem Neutestamentler ist nichts gefährlicher als die Beschränkung auf sein enges Gebiet. “3 1894 wurde er „Correspondirendes Mitglied“ in der Philologisch-Historischen Klasse der Akademie der Wissenschaften zu Göttingen. ${ }^{4}$ Am 2. August 1938 starb der zeitlebens Schwerstbehinderte, über den der Kirchenhistoriker H. Lietzmann bewegend schrieb: „Es liegt auf diesem tapferen Gelehrtenleben von früh an eine erdrückende Kette von Leid."

Für den entschiedenen und - soweit es dem Erblindeten möglich aktiven Gegner des nationalsozialistischen Regimes sollten Nachrufe in der öffentlichen Tagespresse möglichst vermieden werden. ${ }^{6}$

II. Unter Jülichers annähernd 800 Veröffentlichungen auf den Gebieten Neues Testament, Patristik, Textkritik ist heute noch am ehesten sein Werk über die „Gleichnisreden Jesu“ (Bd. I 1886; Bd. II 1899 und Nachdrucke)

$2 \quad$ A. Jülicher, Die Quellen von Exodus I-VII,7. Ein Beitrag zur Hexateuchfrage, Diss. phil. Halle 1880 (Teildruck). Die Fortsetzung: ders., Die Quellen von Exodus VII,8-XXIV,11. Ein Beitrag zur Hexateuchfrage, JPTh 8, 1882, 79-127.272-315.

3 A. Jülicher (s. Anm. 1), 24.

4 Schon zuvor, von P. de Lagarde angeregt, schrieb er seit 1883 in den „Göttinger Gelehrten Anzeigen“ Besprechungen. In vier Jahrzehnten veröffentlichte er dort etwa 50 teilweise umfangreiche Rezensionen.

5 H. Lietzmann, Notizen, ZNW 36, 1937 (erschienen 1938), 293ff., hier: 293 (Zitat).

6 Außer in der Lokalpresse erschien wohl nur anonym ein kurzer Artikel im „Schwäbischen Merkur" am 10.8.1938. Vgl. im übrigen: Glanz und Niedergang der deutschen Universität. 50 Jahre deutscher Wissenschaftsgeschichte in Briefen an und von Hans Lietzmann (1892-1942). Mit einer einführenden Darstellung hg. v. K. Aland, 1979, 132ff. Jülicher war ein politisch interessierter, aber nie parteipolitisch gebundener Zeitgenosse; vgl. A. Jülicher [s. Anm. 1], passim; J.-Chr. Kaiser [s. Anm. 1] (mit auch Überzeichnung von Sachverhalten). Eindeutig kritisch stand er zur Berufungspolitik der Preußischen Hochschulverwaltung gegenüber der Marburger Theologischen Fakultät; dazu seine Streitschrift „Die Entmündigung einer preußischen theologischen Fakultät in zeitgeschichtlichem Zusammenhange“, 1913, in deren Besprechung E. Troeltsch (ThLZ 38, 1913, 401-403) festhält: „Solange Männer wie Harnack und Jülicher an unserer Spitze stehen und ihre warnende Stimme erheben, werden wir den Mut nicht zu verlieren haben" (vgl. auch H.-J. Klauck [s. Anm. 1], 99). 
bekannt, das bis in die jüngste Zeit hinein theologische und auch germanistische Forschung beschäftigt.

Seine „Einleitung in das Neue Testament" (1.2.1894-7'1931 [mit E. Fascher]) darf als Höhepunkt liberaler historisch-kritischer Forschung gewertet werden. Hier wird der Ertrag des durch Carl Weizsäcker (1822-1899) vermittelten Erbes der Tübinger Schule eines Ferdinand Christian Baur (1792-1860) in die nachbaursche Ära, die liberale Epoche Jülichers, eingebracht. Nur Fachkollegen sind Titel und Sachgehalt des Werkes noch gewärtig und wichtig.

Ebenfalls nur Spezialisten des Faches, aber auch Altphilologen, mag die textkritische, bei Jülicher unter dem Namen „Itala“ laufende Überprüfung, Rekonstruktion und Sammlung der lateinischen Textüberlieferung vor deren Bearbeitung durch den Kirchenvater Hieronymus noch von ferne ein Begriff sein. Jülichers, auf 8000 Quartblättern das ganze Neue Testament erschließende Textsammlungen erschienen, überarbeitet für die Evangelien, erst in den Jahrzehnten nach seinem Tode, zum Teil sogar in 2. Auflage. ${ }^{8}$

Schließlich wird der Patristiker Jülicher, der auf dem Gebiet der Alten Kirche die größte Zahl seiner Veröffentlichungen aufweist, nur noch punktuell und gelegentlich herangezogen. Das von Theodor Mommsen angeregte und im persönlichen Austausch mit Jülicher geplante Werk der „Prosopographie“, in dem , über die Zeit Diokletians hinaus bis zu Justinian I." die christlichen Gestalten, nicht nur die „Standesgenossen“ "behandelt werden sollten, erwies sich schon durch die Fülle des Materials als eine letztlich unabschließbare Aufgabe für einen Einzelforscher. ${ }^{9}$

Fast ganz unbeachtet ist heute Jülichers Paulusforschung, obwohl sich gerade an dieser der theologische Umbruch nach dem Ersten Weltkrieg bis hin zur „Dialektischen Theologie“ mit entzündet hat. Auch das zeigt nur an: Jülicher ist in der gegenwärtigen wissenschaftlichen Diskussion nicht mehr im Blick, seine Nachwirkung aber kann schon deshalb nicht so klanglos verhallt sein, weil drei seiner bedeutendsten und engsten Schüler unserer Akademie angehörten: Walter Bauer, Rudolf Bultmann, Hermann Dörries.

Überhaupt ist, wenn man sich der Paulusforschung Jülichers zuwendet, die eigentliche Adresse seiner diesbezüglichen Arbeiten Göttingen. Er war der liberale, historisch-kritische Freund, Förderer, aber auch weiterführen-

Bibliographie darüber seit 1945 bei H. Schmidt (s. Anm. 1), 109; vgl. weiter U. Mell (Hg.), Die Gleichnisreden Jesu 1899-1999. Beiträge zum Dialog mit Adolf Jülicher, BZNW 103, 1999.

8 Bibliographie bei H. Schmidt (s. Anm. 1), 109f.; zu weiteren Einzelheiten H. Lietzmann (s. Anm. 5), 293f.; W. G. Kümmel, Einleitung in das Neue Testament, ${ }^{21} 1983,471 f .589$ (Lit.).

9 A. Jülicher (s. Anm. 1), 32f. [mit Zitaten]. 
de Kritiker der „Religionsgeschichtlichen Schule“. Dies verband ihn über schon lange bestehende Freundschaft auch darin mit Julius Wellhausen, dem er schon als Berliner Student seine einzige „Bekehrung“ verdankte. ${ }^{10}$ Jülicher selbst hat sich bereits in Studientagen ganz bewußt der „liberalen Theologie" zugerechnet.

III. Im Jahr 1907 konstatiert Jülicher, und damit sind wir mitten in seiner Paulusforschung: „ich möchte aber das Bekenntnis aussprechen, daß ich in der geschichtlichen Forschung mich auf keinen anderen Boden stellen kann und will als Bousset und Wrede. "11 Wie aber war diese gemeinsame Grundlage beschaffen? Die Basis war im ganzen die historisch-kritische Forschung in ihrer liberalen Ausprägung mit dem Schwerpunktergebnis in der neutestamentlichen Wissenschaft, die Persönlichkeit Jesu einerseits und die Persönlichkeit des Apostels Paulus andererseits zu erfassen. ${ }^{12}$ Es war durchaus, wie Jülicher 1894 schreibt, Allgemeingut in der Fachwissenschaft des Neuen Testaments, daß die Quellenlage nur hinsichtlich des Paulus „ein leidlich klares Bild von seiner Persönlichkeit“ und „seiner schriftstellerischen Eigenart" erlaubt, und weiterhin, daß diese Gestalt - worauf Jülicher besonderes Gewicht legt - in die Geschichte des Urchristentums eingeordnet und nur aus dieser heraus interpretiert werden kann und muß. ${ }^{13}$ Hinter seinem Paulusverständnis steht in nachhaltiger Vermittlung durch C. Weizsäcker die Paulusdarstellung von Ferd. Christ. Baur. Bei weitreichender Kritik an dessen konstruierter Geschichte des Urchristentums, bei Ablehnung der ihm eigenen Tendenzkritik, hat nach Jülicher gleichwohl der große Tübinger die Grundlage für das geschichtliche Verstehen des Paulus gelegt. „Seit Baur kann die Literarturgeschichte des NT's nicht mehr außerhalb des Zusammenhangs mit der Gesamtgeschichte des Christentums behandelt werden. " 14

Die Rekonstruktion, die jedes antike Schriftstück erfordert und die somit die neutestamentlichen Texte nicht von diesen unterscheidet, ist für Jülicher in seine exegetisch-hermeneutische Grundregel eingebettet: „Denn Worte und Schriften auslegen kann nur, wer die Menschen versteht. "15 Rekon-

\footnotetext{
$10 \quad$ A. Jülicher (s. Anm. 1), 12.

11 A. Jülicher, Paulus und Jesus, RV I / 14, 1907, 8f.

12 Vgl. dazu im Überblick und besonders zu W. Wrede und W. Bousset: O. Merk, Die Persönlichkeit des Paulus in der Religionsgeschichtlichen Schule, in: Biographie und Persönlichkeit des Paulus, hg. v. E.-M. Becker u. P. Pilhofer, WUNT 187, 2005, 29-45.

13 A. Jülicher, Einleitung in das Neue Testament, GThW III / 1, 1.2. 1894, 5; vgl. ebd. \$3: „Der Apostel Paulus“(19-34).

14 A. Jülicher, Einl. (s. Anm. 13), 13.

15 A. Jülicher (a. Anm. 1), 7.
} 
struktion und Interpretation greifen in der Sache des Auslegens ineinander: Nur dann ist Paulus in seiner Persönlichkeit, seinem Wirken und seiner Verkündigung, ja als tragende Gestalt des Urchristentums verstanden. Es wundert darum nicht, daß Rudolf Bultmann in seiner sorgfältigen Nachschrift der Vorlesung von A. Jülicher über „Galater-, Philipper- ,Thessalonicherbriefe “16 so häufig Jülichers Hinweis auf die „Existenz“ aufgreift. Dem entspricht, wenn Jülicher in seinem Artikel „Fleisch und Geist“ (1910) ${ }^{17}$ festhält, „daß das volle Verständnis der Theologie des Paulus und seiner Persönlichkeit abhängt von der Einsicht in seine Auffassung von Fleisch und Geist" (Sp. 911), konkret, daß durch den anthropologischen Rahmen, den Paulus selbst gesetzt hat, die Theologie des Apostels zu erschließen ist. ${ }^{18}$ Jülichers exegetisch-theologischen Bemühungen aber haben dieses Ergebnis: Nur bei Paulus (und Johannes) kann im Neuen Testament von „Theologie“ gesprochen werden. Hier ist der theologische Gipfel, dem dann der Abfall von der Höhe ins christliche gewöhnliche Leben folgt. ${ }^{19}$ Aber das kann nach Jülicher nur gesagt werden, weil Religion und Persönlichkeit Jesu den Ausgangspunkt bilden. ${ }^{20}$

Diese Einsichten hat Jülicher durch den Vollzug genauer Einzelexegese gewonnen. Er liebte die Bezeichnung „Einzelexegese“21, mit der sich für ihn Vers- und Wortanalyse verband. ${ }^{22}$ Philologisch und historisch im Detail arbeiten, heißt bei ihm, „daß ein moderner Theologe, zu dessen Handwerkszeug Wettstein nicht gehört, nicht existiert.“ Die nach Jülicher zu sichtende Sammlung von J.J. Wettsteins Material in dessen „Novum Testamentum graecum“ (Amsterdam 1751/52), „die Hauptquellen Wettsteins“, von denen Jülicher sagt, daß sie „selbstverständlich zu meinem Arbeitsapparat gehören," begegnen fortgesetzt in seiner exegetischen Ausarbeitung

16 Die im Titel genannte Vorlesung hielt Jülicher im W.S. 1905/06. Die Mitschrift befindet sich im Nachlaß von R. Bultmann unter der Nummer Mn 2-3099 in der Universitätsbibliothek Tübingen und wird mit freundlicher Genehmigung von Frau Professorin Antje Bultmann (und Familie Bultmann) benutzt.

17 A. Jülicher, Art. Geist und Fleisch, RGG ${ }^{1}$, Bd. II, 1910, 910-914.

18 Jülichers Kommentar über den Römerbrief (s. Anm. 31) zeigt allerdings den notwendigen Bezug zur "Theologie“, vgl. etwa 254.256.

19 Vgl. A. Jülicher, Die Religion Jesu und die Anfänge des Christentum bis zum Nicaenum, in: P. Hinneberg, Hg., Die Kultur der Gegenwart, Bd. I 4 / 1: Die christliche Religion mit Einfluß der israelitisch-jüdischen Religion, (1906) ${ }^{3} 1922$ mit Nachtrag, 42-131, hier: 86ff.; ders., Art. Sitte und Sittlichkeit: III. Sittlichkeit des Urchristentums, RGG ${ }^{1}$, Bd. V, 1913, 663-683.

A. Jülicher, Die Religion Jesu (s. Anm. 19), 91; vgl. ebd. $54 \mathrm{ff}$.

A. Jülicher (s. Anm. 1), 35.

R. Bultmanns Mitschrift der o.g. Vorlesung (s. Anm. 16) belegt dies eindrücklich. 
der neutestamentlichen Texte. ${ }^{23}$ Von seinen Wort- und Begriffserklärungen kann man sogar behaupten, daß sich in Anlehnung an diese nicht wenige in Walter Bauers „Wörterbuch zum Neuen Testament“ ( $\left.{ }^{2} 1928 ;{ }^{5} 1958\right)$ wiederfinden. Historisch-kritische Eruierung des neutestamentlichen Textbestandes ist für Jülicher die Voraussetzung „liberaler Theologie“, für die Erschließung des Urchristentums ebenso wie für die Erfassung der Persönlichkeiten desselben.

Jülichers Beiträge zur paulinischen Forschung verdanken sich - abgesehen von denen in seiner „Einleitung in das Neue Testament“ und wenigen weiteren Ausnahmen - den Vertretern der „Religionsgeschichtlichen Schule". Von ihnen wurde er zur Mitarbeit in der von W. Bousset und W. Heitmüller gegründeten und herausgegebenen „Theologischen Rundschau“ aufgefordert. Hier erschien sein erster einschlägiger Bericht „Paulinische Theologie". ${ }^{24}$ Weiter baten ihn die Göttinger um die Mitarbeit an ihrer Reihe „Religionsgeschichtliche Volksbücher“, und schließlich haben diese ihn veranlaßt, in ihrem Auslegungswerk „Die Schriften des Neuen Testaments neu übersetzt und für die Gegenwart erklärt ${ }^{\text {“25 }}$ zur Einführung „Die Geschichte des Neuen Testaments" (Bd. 1, 1-30) und weiter die Auslegung des Römerbriefs (Bd. 2, 223-335) zu verfassen. - Auch das von ihnen ins Leben gerufene Lexikon „Die Religion in Geschichte und Gegenwart" (1. Aufl. 1909-1913) wurde von Jülicher u. a. mit Artikeln zur Paulusforschung bedacht.

Es war also tatsächlich die Paulusforschung, die Jülicher vornehmlich mit den Göttingern verband, wenn ihm auch für die Evangelienforschung William Wrede ein maßgebender Gesprächspartner war $^{26}$ und Fragen der

23 Zitate in A. Jülicher, Ein philologisches Gutachten über Phil 2 v. 6, ZNW 17,1916, 1-17, hier: 2f.; zur reichen Lit. über Wettstein vgl. u. a. G. Seelig, Religionsgeschichtliche Methode in Vergangenheit und Gegenwart. Studien zur Geschichte und Methode des religionsgeschichtlichen Vergleichs in der neutestamentlichen Wissenschaft, Arbeiten zur Bibel und ihrer Geschichte, Bd. 7, 2001, bes. 337ff.; vgl. ebd. 56ff.

24 ThR 4, 1901, 187-198 mit dem ernüchternden Fazit: „Mein Gesamteindruck von der hier besprochenen Litteratur ist der schmerzliche, daß selbst auf dem Gebiete des Paulinismus, wo die Einigung der verschiedenen Richtungen am leichtesten erreichbar wäre, wir von einer solchen weiter denn je entfernt sind; beinahe nichts kann als allgemein anerkanntes Ergebnis der wissenschaftlichen Arbeit gelten" (198).

25 1. u. 2. Aufl. (1906ff.) hg. v. Joh. Weiß; 3. Aufl. (1917) hg. v. W. Bousset u. W. Heitmüller; eine „vierte, fast unveränderte Auflage“ erschien 1929.

26 A. Jülicher, Neue Linien der Kritik der evangelischen Überlieferung, Vorträge des Hessischen und Nassauischen theologischen Ferienkurses, Heft 3, 1906, bes. 14-36.67ff.; zu Wrede insgesamt ders., Art. Wrede, William, PRE, Bd. 21, 1908, 506-510; aus neuerer Sicht: W. Zager, Art. Wrede, William, TRE 36, 2004, 337-343; O. Merk, Art. Wrede, W., RGG ${ }^{4}$, Bd. 8, 2005, 1713. 
„Religionsgeschichte“ ihn im Austausch mit der „Religionsgeschichtlichen Schule" lebhaft bewegten. ${ }^{27}$

Ein besonderer Moment kritischen Gesprächs war gegeben, als 1904 das Paulusbüchlein von William Wrede erschien. ${ }^{28}$ Wrede stellte dort einen deutlichen Gegensatz zwischen der Religion und Frömmigkeit Jesu und der Persönlichkeit des Paulus heraus und erklärte aufgrund zahlreicher Nachweisungen Paulus zum zweiten Stifter des Christentum, der nicht einen besseren, sondern einen eher schlechteren Einfluß auf die junge Christenheit ausgeübt habe. Dieser Konzeption mit ihrer unmittelbaren Gegenüberstellung von Jesus und Paulus trat Jülicher in seiner Schrift „Paulus und Jesus" (1907) entgegen. ${ }^{29}$ Im einzelnen lotet Jülicher zunächst das Gegensätzliche „zwischen Paulus und Jesus“ aus (13ff.) und dann den Grad „innerer Verwandtschaft zwischen Paulus und Jesus“ (35ff.; vgl. 12f.), um schließlich eine Einordnung in das "Gesamtbild“ mit der Zuspitzung, ,wer denn der eigentliche Stifter des Christentums gewesen ist", vorzunehmen (12; vgl. 52ff.). „Übereinstimmung und Unterschied“ (52ff.) von Jesus und Paulus erklären sich (in deutlicher Zurechtrückung von Wredes Sicht) aus der verschiedenen heilsgeschichtlichen Situation. „Paulus hat selber als das A und $\mathrm{O}$ seines Evangeliums das Wort vom Kreuz bezeichnet; der Tod Christi ist für ihn die Heilstat ohnegleichen, natürlich sein Tod nur als Voraussetzung für seine Auferstehung “ (25). Somit war der „Ausgangspunkt" der Christologie für Paulus „der auferstandene Mann von Golgatha“ (28). Bei aller von Jülicher konstatierten, auf demselben jüdischen Hintergrund erwachsenen, Jesus und Paulus gemeinsamen und doch je eigenen Frömmigkeit ist gleichzeitig die Verschiedenheit Jesus - Paulus im „Kreuzestod Jesu" gegeben (62). So kann Jülicher - durchaus in gedanklicher Anlehnung an Ferd. Christ. Baur - formulieren: „Die Religion Jesu konnte gar nicht in ihrer ursprünglichen Einfachheit verbleiben, seit Jesus selbst nicht mehr

27 Vgl. etwa A. Jülicher, Besprechung von W. Boussets 'Antichrist' (1895), ThLZ 21, 1896, 373379; ders., Moderne Meinungsverschiedenheiten über Methode, Aufgabe und Ziele der Religionsgeschichte, MakR 5, 1901 [= Rektoratsrede], bes. 6ff. W. Bousset bei O. Merk, Die Persönlichkeit des Paulus (s. Anm. 12.), 34ff. - Daß Wredes Nachweisungen über die Unechtheit des 2 . Thessalonicherbriefs bei Jülicher deutlich nachwirkten,

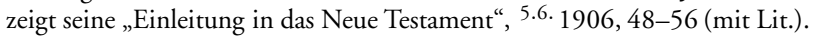

A. Jülicher, Paulus und Jesus (s. Anm. 11). Seitenangaben im Text beziehen sich auf diese Schrift. Zu Jülichers Antwort an Wrede vgl. W.G. Kümmel, Das Neue Testament. Geschichte der Erforschung seiner Probleme, OA III / 3, ${ }^{2} 1970$, 396f. u. Auszüge 397-399; F. Regner, "Paulus und Jesus" im 19. Jahrhundert, Beiträge zur Geschichte des Themas "Paulus und Jesus" in der neutestamentlichen Theologie, Studien zur Theologie und Geistesgeschichte des Neunzehnten Jahrhunderts, Bd. 30, 1977, 191f. [mit harter Kritik an Jülicher, doch ohne den eigentlichen Kern zu treffen]; E. Jüngel, Paulus und Jesus. Eine Untersuchung zur Präzisierung der Frage nach dem Ursprung der Christologie, HUTh 2, ${ }^{2}$ 1961, 9 f.16 [Punkt 2]. 
auf Erden war" (62). Das über die Religion Jesu hinaus Neue ist bei Paulus die Theologie, das Bedenken von Kreuz, „Erlösung, Versöhnung, Rechtfertigung“ (66), in summa: „Das Heil kommt eben aus Jesu Tod“ (67). Daraus ergibt sich: Paulus ist nicht der „zweite Stifter der christlichen Religion“, der "Stiftungstag der christlichen Kirche“ ist vielmehr „der Tag, wo zum ersten Male von Jesusgläubigen der Tod Christi als Heilstatsache begriffen worden ist" (68f.). Hier rundet sich für Jülicher das Sachproblem. Zwischen Jesus und Paulus liegt die, von Wrede in seiner Konzeption nicht beachtete Urgemeinde, und Paulus ist derjenige, der den ersten Jüngern die theologische Panzerung gegeben hat. Mit den Worten Jülichers: „Jesus ist gekommen und hat auf Erden einen neuen Paradiesesgarten angelegt." Doch nach Jesu Tod hatten die Erben einen schweren Stand. „Da kam Paulus und führte um den Garten her die dicken Schutzwälle seiner Theologie auf“ (71): „Paulus hat also seine Theologie nicht an die Stelle der Religion Jesu gesetzt, sondern rings um sie her" (72). Im methodisch äußerst überlegten Ineinander von Rekonstruktion und Interpretation verortete Jülicher Jesus und Paulus im Urchristentum und verweist so in seiner Entgegnung auf Wrede Religion und Theologie in ihren einander bedingenden historischen Rahmen. Unverkennbar ist dabei, daß Jülicher für seine Konzeption den Aufriß Ferd. Christ. Baurs „Vorlesungen über neutestamentliche Theologie" für seine Fragestellung nachbedenkt und auswertet. ${ }^{30}$

Hier ist in Kürze Jülichers Römerbriefauslegung anzuschließen und damit mehr summarisch zu Einzelheiten seiner exegetischen Einsichten zu kommen: ${ }^{31}$

a) Daß Paulus sich in seinen Briefen als Schriftsteller von Rang erweist, läßt sich besonders bezüglich des Römerbriefs behaupten. Diese Sicht in der ersten Auflage seiner „Einleitung in das Neue Testament“ (1894) schränkt Jülicher allerdings bis zur 7. Auflage (1931) immer stärker dahingehend ein, daß die paulinischen Briefe dem Apostel aufgenötigte Gelegenheitsschreiben seien, was ihnen aber ihre literarische Bedeutsamkeit nicht nehme.

b) Der Römerbrief - und das ist zu Jülichers Zeit keineswegs in Fachkreisen anerkannte Meinung - hat ein durchgängiges Thema: die Rechtfertigung. Die Disposition des Schreibens muß dem Rechnung tragen,

30 Ferd. Christ. Baur, Vorlesungen über neutestamentliche Theologie, hg. v. Ferd. Friedr. Baur [1864]. Mit einer Einführung zum Neudruck v. W.G. Kümmel, 1973, bes. die Abschnitte „Übergang der Lehre Jesu zu der der Apostel“ u. „Die Auferstehung Jesu“ (122-127), sehr bewußt zwischen 45-121 u. 128ff. stehend.

31 A. Jülicher, Der Brief an die Römer, SNT, Bd. $2,{ }^{3}$ 1917, 223-335 (Seitenangaben im Text nach dieser Ausgabe). 
wobei insbesondere Kap. 9-11, die Israelkapitel, dem Grundthema in 1,16f. zugehören (225.292). Kap. 5-8 bilden ebenfalls eine Einheit, während Kap. 12-15,13 insgesamt lockerer zum Briefkorpus stehen (309ff.). „Kurz, wie zuletzt alles in Röm. 12-14, ein Ideal“ (325), aber gleichwohl werden ,usuelle“ und „aktuelle“ Weisungen (ausdrücklich von Jülicher so charakterisiert [310.319]) herausgearbeitet. - Kap. 16 bleibt in seiner Zugehörigkeit zum Römerbrief offen, die Verse in Kap. 16,25-27 (Doxologie) sind sekundär (335 u.ö.).

c) Die Abfassung des Schreibens muß nach Röm 15,26 „hinter 2. Kor 8.9“ (die Kollektenkapitel) fallen. ${ }^{32}$

d) Eingehend befaßt sich Jülicher mit "Glossen“ im Römerbrief, wobei er nahezu alle Stellen anführt und bespricht $(2,1.16 ; 7,25 ; 8,1$ [224f.240.279], die später R. Bultmann in einem berühmt gewordenen Aufsatz behandelt. ${ }^{33}$ Hinzu kommen erhellende textkritische Erörterungen, die auch ein Laie verstehen kann.

e) Erhebliche Bedeutung mißt Jülicher evtl. Traditionsstücken bei. Über Röm 1,3f.; 8,3 (vgl. z. St. u. 281) hinaus zeigt er - ohne sich für ein Traditionsstück zu entscheiden -, daß Röm 3,24ff. den Sprachgebrauch der Urgemeinde, nicht aber den des Paulus etwa in 3,21-23 bietet. Jülicher hat in der Sache erkannt, was wir in Röm 3,25f. heute in der Regel als Traditionsstück bezeichnen.

f) In Röm „7,14ff. schildert Paulus den vorchristlichen Menschen, wie er, vom christlichen Standpunkt her gesehen, erscheint" (279; vgl. 278 u.ö). Jülicher hat hier, auch durch die große Zurückhaltung gegenüber einer psychologischen Ausdeutung dieser und anderer Stellen (vgl. 296.302), exegetisch-theologisch einer Sicht vorgearbeitet, die, allseitig begründet durch W.G. Kümmel, weithin die Forschung zu diesem Abschnitt im 20. Jahrhundert bestimmt hat. ${ }^{34}$

g) „Das Gesetz ist seit Christus ungültig“ (299), es muß aber im Gesamtzusammenhange der Theologie des Paulus - nicht zuletzt im Röm gesehen werden (272ff.): „Wir Heutigen haben die Empfindung, daß es für den Segen des Gesetzes, auch gerade des jüdischen, keinen gewaltigeren Zeugen gibt als den Heidenapostel“ (274).

\footnotetext{
32 Daß chronologisch nach Röm. noch Philipperbrief, Philemonbrief, Kolosserbrief einzuordnen sind, zeigt Jülicher in seiner „Einleitung in das Neue Testament“, zuletzt ${ }^{7} 1931$.

33 R. Bultmann, Glossen im Römerbrief (1947), in: ders., Exegetica. Aufsätze zur Erforschung des Neuen Testaments, ausgew., eingel. u. hg. v. E. Dinkler, 1967, 278-284 (mit ausdrücklichem Hinweis auf Jülicher [279 Anm. 1]).

34 W. G. Kümmel, Römer 7 und die Bekehrung des Paulus, UNT 17, 1929, mit vielfachem Bezug auf Jülicher, bes. zu dem zitierten Ergebnis, vgl. u. a. 121.126.
} 
h) Zur eschatologischen Sicht des Paulus im Röm führt Jülicher einerseits aus: „Man darf nicht übersehen, daß Röm 2,6-11 nicht bestimmt sind, die Vorstellungen des Apostels Paulus über das Weltgericht und über das letzte Schicksal des Menschen zu entwickeln" (238; auch teilweise in der Auslegung zu Röm 9-11 relevant). Andererseits bestreitet er nicht die Bedeutung der Nähe des Weltendes im Glauben des Paulus sowie deren Auswirkung, sittliche Energien freizusetzen (zu Röm 13,11.12 [319]). Aber eine wirkliche Verankerung der Eschatologie im Denken des Paulus fehlt bei Jülicher.

So viele wichtige Beobachtungen dieser Kommentar enthält und so gewiß exegetische Probleme seiner Zeit in die Ausführungen eingegangen sind, Jülicher hat sie in seiner weiterführenden Auslegung auf den Punkt gebracht. ${ }^{35}$ Der Kommentar ist durchgehend ein Zeugnis „liberaler Theologie“. ${ }^{36}$ Der Römerbrief ist „die Haupturkunde der paulinischen Religion“, er ist des Paulus „Glaubensbekenntnis“ (224f.). „Das Evangelium von Christi Heilswerk bietet Gerechtigkeit und ewiges Leben dar jedem gläubigen Menschen“ (244), und die „Rechtfertigung“ bzw. „die Gerechtmachung“ ist für den Christen ein Prozeß der „Entscheidung, die nicht mit einem Schlag fertig ist, sondern wie eine Art von Verwandlung des Menschen aus dem Fleischlichen ins Göttliche über sein ganzes ferneres Erdenleben hin andauert“ (246f.). Das Verständnis des Reiches Gottes in der „liberalen Theologie" als einer inwendigen, wachsenden Größe im Menschen wird von den Evangelien her auf die paulinische Rechtfertigung übertragen. - Aber es gilt ebenso, und das ist nicht nur für „liberale Theologie“ stehend: „Der Glaube des Christen ist für Paulus das Vertrauen auf die durch Jesu Christi Sterben und Auferstehung von Gott gewirkte Vergebung unserer Sünden und auf unsre Umwandlung aus Sündern in Gerechte unter der einzigen Voraussetzung, daß wir dieses Vertrauen haben: ganz wie 3,21-30“ (255).

35 Wie zurückhaltend der Autor über seine eigenen Leistungen dachte, zeigt A. Jülicher, Einleitung in das Neue Testament (s. Anm. 13), Vorwort (S. V): „daß beinahe alles, was ich hier vortrage, durch die treue Arbeit ganzer Generationen zusammengebracht und nicht von mir entdeckt worden ist, weiss wohl Jedermann. Prioritätsansprüche werde ich aufgrund dieses Buches gegen Niemanden erheben."

„Liberale Theologie“ war zu Jülichers Zeit durchaus vielschichtig in ihren Ausprägungen und auch im Bereich der Bibelwissenschaft äußerst different; vgl. geradezu resigniert H. Mulert, Art. Liberalismus: II. Liberalismus und Kirche, RGG ${ }^{1}$, Bd. III, 1912, 2107ff., hier 2109: "Von theologischem L.[iberalismus] oder liberaler Theologie zu reden, wird besser vermieden“; eindeutiger schon A. Meyer, Art. Bibelwissenschaft: II. Neues Testament, RGG ${ }^{1}$, Bd. I, 1909, $1212 \mathrm{ff}$., bes. $1219 \mathrm{ff}$., $1227 \mathrm{ff}$. Aus neuerer Sicht im weitgefaßten Überblick vgl. M. Jacobs, Art. Liberale Theologie, TRE 21, 1991, 47-68; insgesamt der Zeit Jülichers exegetisch u. bibelwissenschaftlich näher: W. Zager, Liberale Exegese des Neuen Testaments. David Friedrich Strauß - William Wrede - Albert Schweitzer - Rudolf Bultmann, 2004. 
Eine liberale Leitlinie kennzeichnet die ethischen Partien des Briefes. Das gilt ebenso für Kap. 6 wie für 12.1ff., so gewiß Jülicher in einem Exkurs festhält: „Seine ganze Ethik, beinahe seine ganze Religion hat Paulus auf den Geist gegründet" (276). Aber Jülicher kann es auch so formulieren: "Gottes Wille ist einfach das, was ein Mensch vor seinem Gewissen als gut rechtfertigen kann. So ist die Versöhnung von Religion und Sittlichkeit gefunden; die einzige religiöse Pflicht, die im Christentum übrig bleibt, ist der Wandel im Stil der zukünftigen Welt; fromm sein heißt nichts weiter als gut sein im Denken und Tun“ (310).

Jülichers Römerbrief-Kommentar ist auch durch seine geschliffene Sprache und Formulierung sowie durch seine dichte Argumentation eine herausragende Auslegung seiner Zeit gewesen. ${ }^{37}$

R. Bultmanns offenbar sehr genau nachgezeichnete, o. g. Vorlesung Jülichers über den Galaterbrief, den Philipperbrief und die beiden Thessalonicherbriefe im W.S. 1905/06 - im Manuskript Bultmanns 201 Seiten -ist die einzige Vorlesung dieser Art, denn die Vorlesungsmanuskripte Jülichers sind offensichtlich nicht erhalten geblieben. ${ }^{38}$

Zwei Hinweise seien aus der Vorlesungsnachschrift Bultmanns für Jülichers Paulusverständnis angeführt: ${ }^{39}$

a) Zu Gal 2,9 stellt Jülicher in der Nachschrift Bultmanns über die Aufteilung der Missionsgebiete fest: „Das Princip sagt natürl.[ich] nicht, daß $\mathrm{Pls}$ [= Paulus] nie einen Juden, Pt. [= Petrus] nie einen Heiden bekehren sollte. Das Princip war auf die Dauer nicht haltbar" (MS, 37). Jülicher wollte dies dahin verstanden wissen, wie der Paulusteil seiner „Einleitung" zeigt, daß Petrus durchaus auch in Korinth und anderswo gewirkt haben könnte. ${ }^{40}$

b) Wichtiger ist die mittelbar sich mit W. Wrede befassende Auslegung des Antiochiakonflikts in Gal 2,11-14, denn Jülicher meint, daß der Abschnitt bis 2,21 reicht, und zeigt auf, daß die Rechtfertigungslehre bereits in diesem Konflikt bestimmend sei (MS, 43ff.). Mag diese hier noch

37 Es wundert darum nicht, daß Jülicher die Kommentierung des Römer- u. des Galaterbriefs in „Meyers kritisch-exegetischem Kommentar" angetragen wurde. Diese hätte er gerne vorgenommen. Er gab diesen Auftrag nicht nur wegen seiner Erblindung auf, sondern auch, weil er meinte, dies könnten andere ebenso gut, während die textkritische Arbeit an der "Itala“ seiner in Jahrzehnten erworbenen Spezialkenntnisse weiterhin bedürfe.

38 Vgl. o. Anm. 16. Recherchen haben bisher nur dieses Ergebnis gebracht; im übrigen ist der Nachlaß Jülichers in der Marburger Universitätsbibliothek unter Nummer MS 695. Zur Beschreibung des Nachlasses vgl. H.-J. Klauck (s. Anm. 1), 149.

39 Angaben / Belege sind mit MS u. Seitenzahl gekennzeichnet.

40 A. Jülicher, Einleitung in das Neue Testament. Neubearbeitung in Verbindung mit E. Fascher ${ }^{7} 1931,37 \mathrm{ff}$. u.ö. $73 \mathrm{ff}$. (passim). 
nicht die Breite der später bei Paulus vorliegenden Argumentation tragen, so ist doch erkannt, daß nicht Wredes Sicht von der Rechtfertigung als einer sich erst im Zusammenhang der galatischen Auseinandersetzung gebildeten Kampfeslehre ${ }^{41}$ gelte, sondern die Rechtfertigung bereits in die früheste, schon antiochenische Zeit paulinischer Lehre gehöre. Aus Bultmanns Mitschrift ergibt sich als Jülichers ${ }^{42}$ Sicht zu Gal 2,16: „Ob $\operatorname{Ptr}[=$ Petrus] u.[nd] die anderen Christen in Antiochien das Verständnis für die Antithese im Denken des Pls [Paulus] hatten, ist sehr fraglich. Wohl stets glaubte man in der Kirche durch Werke und durch den Glauben die Seligkeit zu erlangen. Sollte das Christentum eine selbständige Religion werden, sollte es frei werden vom Judentum, so war es notwendig, daß die Antithese erga nomou u.[nd] pistis gestellt wurde. Auf die Werke des jüd.[ischen] Gesetzes mußte einmal principiell verzichtet werden. Diese Antithese gestellt zu haben, ist die histor.[ische] Bedeutg [tung] des Pls [= Paulus] " (MS, 45), ${ }^{43}$ und diese Antithese auch im Konfliktfall existentiell bewährt zu haben, zeigt den Theologen Paulus. Dazu als Zitat Jülichers von Bultmann eingebracht: „”Auf die selbst verdiente Gerechtigkeit verzichten wir u. [nd] eignen uns die Gerechtigkeit an, die aus Gnaden dem Glauben geschenkt ist““ (MS, 45).

Auch diese Vorlesung zeigt: Jülicher weiß sich als Historiker. Neutestamentliche Wissenschaft und ihre Methodik gehören wie die Kirchengeschichte zur historischen Disziplin in der Theologie, doch unvermindert in den Grenzen der bedingenden Voraussetzung, daß „auszulegen“ nur vermag, „wer die Menschen versteht. “44 Und dem dient ein die Paulusforschung einbeziehendes, aber sie übergreifendes Anliegen Jülichers.

Es geht ihm, wie er in seiner Rektoratsrede (1901) erläutert, um die ”Demokratisirung ““ [sic!] als Aufgabe der historischen Disziplin in der Theologie, ${ }^{45}$ respektive der Kirchengeschichte im besonderen. „Demokratisirung" war natürlich im damaligen Kaiserreich ein nicht gern gehörtes Wort. Der von Jülicher aus einer belanglosen Untersuchung seiner Zeit ent-

$41 \quad$ So W. Wrede (s. Anm. 28).

42 Dieser ist Bultmann offenbar selbst gefolgt: vgl. ders., Das Problem der Ethik bei Paulus (1924), in: ders., Exegetica (s. Anm. 33), 36-54, hier: 53 Anm. 11.

43 Vgl. ebd., MS, 45f.: „Ptr [Petrus] war sich natürl.[ich] nicht bewußt, durch das Aufgeben der Tischgemeinschaft die Gerechtigkeit aus den Werken vertreten zu haben. Aber dies lag in der Konsequenz des Verhaltens des Petr [= Petrus], u.[nd] Pls [= Paulus] sah vielleicht schon die Folgen in der heidenchristl.[ichen] Gemeinde. So behandelt Pls [= Paulus] die Frage mit Recht als Principienfrage."

44 A. Jülicher (s. Anm. 1), 7.

45 A. Jülicher, Moderne Meinungsverschiedenheiten (s. Anm. 27), bes. 17ff. (Seitenangaben im folgenden innerhalb des Textes beziehen sich auf diese Rede). 
lehnte Begriff wurde ihm in der Sache zu einer zentralen Fragestellung. Die in der „liberalen Theologie“ im neutestamentlichen Bereich so bedeutsame Erfassung der Persönlichkeit, wie derjenigen Jesu oder der des Apostels Paulus, muß geweitet werden im Blick auf die Menschen allgemein, z. B. auf die Menschen in der Bildhälfte der Gleichnisse Jesu; es müssen die Leser der paulinischen Briefe einbezogen werden, es müssen die angeredeten Gemeindeglieder in ihrem Dasein, ihrem Leben, mit ihren Sorgen erfaßt werden. Jülicher gleitet hier nicht in eine freischwebende, isolierte sozialgeschichtliche Forschung ab, sondern er will aufzeigen: „In der Regel wird auch damals die Umwandlung bestenfalls eine allmählich fortschreitende gewesen sein; der Mensch, der sich seines Glaubens wegen als geistlicher Mensch fühlte, hörte darum noch lange nicht auf zu bleiben, was er gewesen war" (19f.; vgl. 22f.). Jülicher weiß um den - wie er es nennt - „aristokratischen Charakter der christlichen Religion", und er weiß, daß ,"Demokratisirung" im gewissen Sinn Verweltlichung der Kirchengeschichte" ist und daß dieses die Welt-in-den-Blick-Nehmen auch in den Konsequenzen für die exegetische Arbeit am Neuen Testament zu bedenken ist und zu beherzigen bleibt (23 u. ö.). Die Konsequenzen aber reichen für ihn noch weiter. Sie betreffen die Theologie als Wissenschaft im Gefüge der Universität: „Entschlossen müssen wir die Schranken der theologischen, der kirchlichen, der erbaulichen Litteratur überschreiten“" (23). Jülicher zählt im weiteren auf, welch großes Feld sich hier für den Theologen und auch eigens für den Exegeten erschließt in der Mitarbeit daran: „So häufen wir in stiller, bescheidener ... Arbeit Bausteine für eine Geschichte, zwar nicht der offiziellen Kirche ${ }^{46}$, manchmal eher der Unkirchlichkeit, doch immer des Christentums, Zeugnisse für seine Elasticität, für seine unerschöpfliche Kraft, sich anzupassen, sich umzubilden, sich zu den Niedrigen herabzulassen und das Gewöhnliche zu adeln" (23). Theologie als Wissenschaft hat sich in die Welt der Wissenschaften und im Kontext der Religionen einzubringen (23f.), und die Bibelwissenschaft hat daran einen gewichtigen Anteil.

Es war deshalb für Jülicher, der in solcher Breite und Weite, der so gesamttheologisch auch über seine engere Fachwissenschaft hinaus dachte, schmerzlich, zu erleben, wie eine veränderte, durch den Ersten Weltkrieg aufgewühlte Generation über diese Arbeit historisch-kritischer liberaler Forschung hinwegging. Karl Barths Römerbrief in seiner 1. Auflage 1919 war von anderem Geiste. Jülicher hat das Wollen der jüngeren Theologen, die zum Teil seine eigenen Hörer und Schüler waren, durchaus gesehen

46 Wie hier Jülicher von „Rechtgläubigkeit“ - auch Orthodoxie und geradezu Ketzerei - des weiteren spricht (23f.), läßt rückblickend Stichworte in seines Schülers Werk Walter Bauer, Rechtgläubigkeit und Ketzerei im ältesten Christentum, BHTh 10, 1934, erkennen. 
und auch anerkannt in seiner im übrigen äußerst kritischen Besprechung dieses Werkes. ${ }^{47}$ Es fehle den Ausführungen Barths die solide historischkritische Forschung. Vor allem aber vermißt Jülicher in Barths Kommentar die Ehrfurcht vor dem Text des Paulus. Und daß sich einer neben, vielleicht sogar über Paulus zu stellen wage, konnte er nur als unangemessenen geistigen Hochmut werten. In einem umfangreichen handschriftlichen Brief aus Safenwil vom 14. Juli 1920 an Jülicher ${ }^{48}$ hat Barth um Verständnis seiner und seiner theologischen Freunde Situation geworben und Mißverständnisse auszuräumen versucht. Barth will da anfangen, wo seiner Meinung nach Jülichers Auslegung des Römerbriefs endet, wobei freilich die auf Jülicher gemünzte Frage, „bei welchem Grad der Unbeteiligtheit am Objekt die Eintrittsberechtigung in den Tempel der Wissenschaft anfängt? “49), an den Falschen gerichtet ist. Jülicher hat auf diesen Brief - soweit ermittelbar - nicht geantwortet, wohl aber die zweite, völlig umgearbeitete Auflage von Barths Römerbrief, ebenfalls positive Momente benennend, sehr kritisch besprochen ${ }^{50}$ und abschließend festgehalten: „Aber, daß Barths Versuch, den Paulus mit Beschlag zu belegen für eine Weltanschauung, die schon seinem naiven Schrift- (und dadurch in gewissem Sinn doch wieder Buchstaben-)glauben ins Gesicht schlägt, der Hybris eines Pneumatikers entspringt und nicht aus nüchterner Wissenschaft, ist das letzte Wort, das ich über einen Römerbrief Barths sagen werde“ (542).

Es war in gewisser Hinsicht eine Tragik, daß in der Krisenzeit für die Kultur wie für die Wissenschaft nach dem Ersten Weltkrieg Jülicher und Barth nicht zueinander finden konnten, obwohl beide von ihren je eigenen Voraussetzungen her nicht nur ein Stück weit Recht hatten.

R. Bultmann hat (1924) in seinem Aufsatz „Die liberale Theologie und die jüngste theologische Entwicklung ${ }^{\text {“51 }}$ die Problematik im Grundsätzlichen aufgedeckt und betont: „Es ist kein Zufall, daß die jüngste theolo-

$47 \quad$ A. Jülicher, Ein moderner Paulusausleger (1920) in: Anfänge der dialektischen Theologie, hg. v. J. Moltmann, ThB 17 / I, ${ }^{5} 1985,87-98$.

48 In Jülichers Nachlaß in der Univ. Bibliothek Marburg, MS 695, 20, mit freundlicher Genehmigung von Herrn Dr. Reifenberg, Univ. Bibl. Marburg, angeführt und zitiert; vgl. auch den Teildruck in H. Graß, Karl Barth und Marburg. Rede zur Eröffnung der Karl Barth-Ausstellung in der Universitäts-Bibliothek am 9.1.1971, hg. v. Marburger Universitätsbund, 1971, 6., u. Bezugnahme bei W. G. Kümmel (s. Anm. 1), 242f. Zu zahlreichen Einzelaspekten der damaligen Diskussion vgl. O. Merk, Karl Barths Beitrag zur Erforschung des Neuen Testaments (1989), in: ders., Wissenschaftsgeschichte und Exegese. Ges. Aufs. zum 65. Geb., hg. v. R. Gebauer, M. Karrer u. M. Meiser, BZNW 95, 1998, 187-211, hier: 193-201 (Lit.).

49 Brief Barths S. 4, freilich auf Jülichers Vorwurf eines „Pneumatikerhochmuts“.

50 A. Jülicher, Bespr. von Barth, K., Der Römerbrief, 2. Auflage in neuer Bearbeitung, (XVII, 523 S.) 1922 , ThLZ 47, 1922, 537-542.

51 R. Bultmann, Die liberale Theologie und die jüngste theologische Entwicklung, in: ders., Glauben und Verstehen, Ges.Aufs. Bd. I, ${ }^{4} 1961,1-25$. 
gische Bewegung aus der liberalen Theologie geboren ist" (1), und es ist kein Zufall, daß er - selbst der liberalen Theologie vielfach verpflichtet ${ }^{52}$ in einer tiefgreifenden Besprechung des Römerbriefs, 2. Aufl. 1922, das Anliegen Barths treffend darlegt mit der ausdrücklichen Feststellung: „daß ich mich übrigens sowohl was die historisch-philologische Erklärung betrifft, wie was die sachliche Beurteilung des Römerbriefs und des Paulus angeht, in hohem Maße dem anschließe, was Jülicher zur ersten Auflage ausgeführt hat, wird Barth teils dem Gesagten entnehmen, teils sich selbst sagen. ${ }^{\text {" } 53} \mathrm{Da}$ mitten in der hohen Zeit der „Dialektischen Theologie“ eine 4. Auflage seiner Römerbriefauslegung 1929 erschien, war für Jülicher eine stille Freude.

Jülicher sah, daß eine neue Epoche im theologischen Denken heraufgezogen war, aber sein Rücktritt vom Lehramt 1923 war keine Resignation. Er hat sich - wie er 1922 schreibt - „nicht verblüffen lassen“ von K. Barth und anderen, wohl aber selbstkritisch zu bedenken gegeben: „ihre Erfolge lassen doch merken, daß an dem hergebrachten Betrieb etwas Wesentliches fehlen muß: man hat die Texte zu wenig in ihrer Eigenart, als religiöse Offenbarungen, gewürdigt, zu sehr auf das Wie und Woher geachtet, nicht genug auf das Was und hat manchmal auch wohl nicht mutig genug gezwungene Deutungen aufgegeben ". ${ }^{44}$ Jülicher blieb ein Lernender. ${ }^{55}$ Aber es keimte in ihm hinsichtlich der Paulusforschung - hier seine Göttinger Freunde gedanklich einschließend - eine Rück- wie Neubesinnung auf den von ihm so häufig mittelbar wie unmittelbar herangezogenen Ferd. Christ. Baur: „Ob wohl die Zeit naht, wo neben der Konstruktion der modernen Religionshistoriker und ihres Widerparts die von Ferd. Christ. Baur wieder zu Ehren gelangt? Gewiß bedarf sie erheblicher Einschränkungen, gewiß müssen bedeutsame Wahrheitsmomente aus der Geschichtsauffassung der Boussetschen Schule mit ihr in Verbindung gebracht werden. Aber ganz an

52 Ebd. (s. Anm. 51), 2 : „Wir, die wir von der liberalen Theologie herkommen, hätten keine Theologen werden oder bleiben können, wenn uns in der liberalen Theologie nicht der Ernst der radikalen Wahrhaftigkeit begegnet wäre.“

53 R. Bultmann, Karl Barths „Römerbrief“ in zweiter Auflage (1922) in: Anfänge der dialektischen Theologie (s. Anm. 47), 119-142, hier: 141 Anm. 4 [Zitat]; im übrigen vgl. die eingehende Analyse von M. Dreher, Rudolf Bultmann als Kritiker in seinen Rezensionen und Forschungsberichten. Kommentierende Auswertung, Beiträge zum Verstehen der Bibel, Bd. 11, 2005, 151-186.

54 A. Jülicher, Die Religion Jesu (s. Anm. 19), [Nachtrag] 131.

55 Vgl. auch seine Diskussion im Anschluß an einen Vortrag v. E. Thurneysen in Marburg im Februar 1924 mit dem Vortragenden, mit M. Rade, R. Bultmann u. M. Heidegger; Hinweis in: Anfänge der dialektischen Theologie, Teil II: R. Bultmann, F. Gogarten, E. Thurneysen, hg. v. J. Moltmann, BTh 17 / II, ${ }^{4} 1987,220$. 


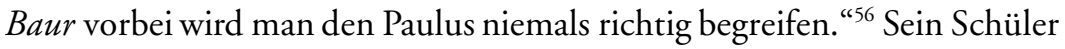
R. Bultmann hat dies dann eindrücklich aufgegriffen. ${ }^{57}$

Brechen wir ab. Über Jülicher ist manches ehrende Wort einst gesagt und geschrieben worden. Zitiert sei nur eines. Franz Overbeck hat sich privat notiert: „Wer etwas lernen und nicht gerade sich unterhalten will, wird viel besser an Jülicher gewiesen, als an Harnack." Und er fährt an weiterer Stelle fort: „Mit Jülicher ist viel eher auf einem guten und festen Grund zu wertvollen Erkenntnissen zu kommen. ${ }^{158}$ Lassen wir Overbecks Beurteilung Harnacks auf sich beruhen. Lassen wir ebenso das leicht nachweisbar Zeitbedingte und exegetisch aus heutiger Sicht Überholte in Jülichers Werk auf sich gestellt. Festzuhalten aber ist: In unserer Gegenwart, in der theologische Wissenschaft so häufig auf Gedanken und Einsichten der „liberalen Theologie" zurückgreift und diese nicht selten als eigene neue Erkenntnisse ausgibt, bleibt Adolf Jülicher ein kritischer Lehrmeister. Er ist ein Theologe, dessen innovative, zur selbständigen Urteilsfindung anregende Arbeiten auch in der Paulusforschung weiterhin allen Nachbedenkens wert sind.

56 A. Jülicher, Die Religion Jesu (s. Anm. 19), Nachtrag 131e (sic!).

57 Vgl. R. Bultmann, Zur Geschichte der Paulusforschung, ThR, N.F. 1, 1929, 26-59; vgl. dazu die Besprechung von M. Dreher (s. Anm. 53), $373 \mathrm{ff}$.

58 F. Overbeck, Christentum und Kultur. Gedanken und Anmerkungen zur modernen Theologie, hg. v. C. A. Bernoulli, 1919, 211f.; weitere Belege für die Hochschätzung Jülichers bei H.-J. Klauck (s. Anm. 1), 99 f. 